tion, the mice have normal body weight, adiposity, locomotor activity, and glucose homeostasis, similar to the findings in the patient.

The data from these transgenic mice support the authors' supposition that nonclassical regulation of estrogen receptor $\alpha$ is sufficient to protect against the obesity-metabolic syndrome phenotype associated with a total loss of activity of estrogen receptor $\alpha$ but is not sufficient to rescue the infertility phenotype. We offer the murine findings as support of the authors' initial claims, and we believe these findings further implicate a differential role for classical versus nonclassical activity of estrogen receptor $\alpha$ in the regulation of energy and reproductive homeostasis.

Deborah J. Clegg, Ph.D.

Biff F. Palmer, M.D.

University of Texas Southwestern Medical Center

Dallas, TX

deborah.clegg@utsouthwestern.edu

No potential conflict of interest relevant to this letter was reported.

1. Quaynor SD, Stradtman EW Jr, Kim H-G, et al. Delayed puberty and estrogen resistance in a woman with estrogen receptor $\alpha$ variant. N Engl J Med 2013;369:164-71.

2. Glidewell-Kenney C, Hurley LA, Pfaff L, Weiss J, Levine JE, Jameson JL. Nonclassical estrogen receptor alpha signaling mediates negative feedback in the female mouse reproductive axis. Proc Natl Acad Sci U S A 2007;104:8173-7.

3. Park CJ, Zhao Z, Glidewell-Kenney C, et al. Genetic rescue of nonclassical $\mathrm{ER} \alpha$ signaling normalizes energy balance in obese Er $\alpha$-null mutant mice. J Clin Invest 2011;121:604-12.

DOI: $10.1056 / N E J M c 1310364$

THE AUTHORS REPLY: Similarities between the correspondents' transgenic mice and our patient with estrogen resistance (with both showing impaired classical estrogen signaling) are important because they reaffirm the relevance of murine models in the study of human disease. Studies in both Esr1 knockout and knock-in transgenic mice indicate that the function of estrogen receptor $\alpha$ not only is important in reproduction but also is key in energy regulation. ${ }^{1,2}$ These important findings, which would be challenging to study in humans, have confirmed translational relevance, as shown in our estrogen-resistant patient. The prevalence of ESR1 mutations in the general population is unknown, but given the murine and human phenotypes, more persons with such mutations may be identified in the future. Our findings confirm the existence of ESR1 mutations in women, which contradicts previous assertions that the absence of function of estrogen receptor $\alpha$ would be lethal in women. ${ }^{3}$ Together, the mouse models and long-term follow-up of our patient, as well as newly identified estrogen-resistant patients, will be important in the study of the longitudinal consequences of absent classical estrogen signaling.

Samuel D. Quaynor, M.S.

Georgia Regents University

Augusta, GA

Derek A. Schreihofer, Ph.D.

University of North Texas Health Science Center at Fort Worth Fort Worth, TX

Lawrence C. Layman, M.D.

Georgia Regents University

Augusta, GA

lalayman@gru.edu

Since publication of their article, the authors report no further potential conflict of interest.

1. Glidewell-Kenney C, Hurley LA, Pfaff L, Weiss J, Levine JE, Jameson JL. Nonclassical estrogen receptor alpha signaling mediates negative feedback in the female mouse reproductive axis. Proc Natl Acad Sci U S A 2007;104:8173-7.

2. Park CJ, Zhao Z, Glidewell-Kenney C, et al. Genetic rescue of nonclassical $\mathrm{ER} \alpha$ signaling normalizes energy balance in obese Er $\alpha$-null mutant mice. J Clin Invest 2011;121:604-12.

3. Smith EP, Boyd J, Frank GR, et al. Estrogen resistance caused by a mutation in the estrogen-receptor gene in a man. $\mathrm{N}$ Engl J Med 1994;331:1056-61. [Erratum, N Engl J Med 1995;332:131.]

DOI: $10.1056 /$ NEJMc1310364

\title{
Acute High-Altitude Illnesses
}

TO THE EDITOR: Bärtsch and Swenson (June 13 issue $)^{1}$ refer to our study assessing risk factors for severe high-altitude illnesses ${ }^{2}$ and conclude that physiological measurements "add little to the discrimination provided by patient . . . history." In our prospective study involving clinical and physiological evaluation of a large cohort of persons before their stay at high altitudes, ${ }^{2}$ we found that adding physiological variables to a multivariate prediction model that included clinical risk factors for severe high-altitude illnesses improved the accuracy of the prediction (the C-statistic increased from 0.81 to $0.88 ; \mathrm{P}<0.001$ ). Moreover, a great proportion of persons who present for a consultation with a specialist in mountain medicine (45\%) have never been to high 
altitudes before, ${ }^{2}$ so clinical information about a history of severe high-altitude illnesses is not available; this reinforces the usefulness of measuring the ventilatory response to hypoxia. ${ }^{3}$ The test to assess the response to hypoxic conditions during exercise is inexpensive ( $€ 100$, or $\$ 135$ in 2013 U.S. dollars), reproducible, ${ }^{4}$ and brief $(<20$ minutes). In contrast to the authors' assertion that "there are currently no reliable tests to predict . . . high-altitude illnesses," we recommend this test for persons who are preparing to travel to high-altitude regions.

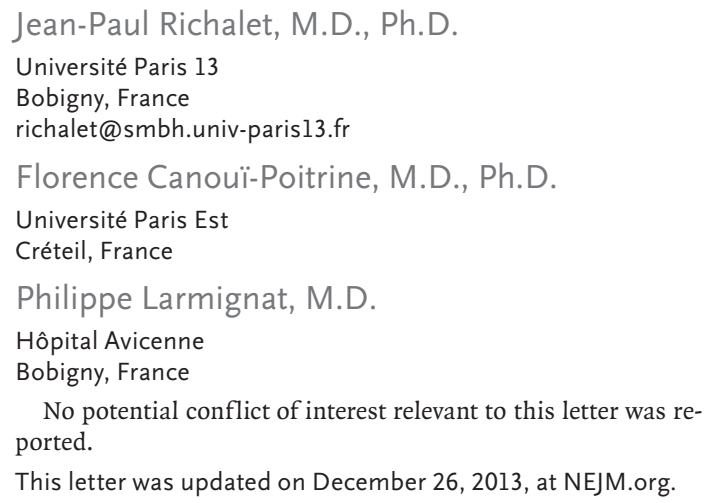

1. Bärtsch P, Swenson ER. Acute high-altitude illnesses. N Engl J Med 2013;368:2294-302.

2. Richalet JP, Larmignat P, Poitrine E, Letournel M, CanouïPoitrine F. Physiological risk factors for severe high-altitude illness: a prospective cohort study. Am J Respir Crit Care Med 2012;185:192-8.

3. Lhuissier FJ, Canouï-Poitrine F, Richalet JP. Ageing and cardiorespiratory response to hypoxia. J Physiol 2012;590:5461-74.

4. Lhuissier FJ, Brumm M, Ramier D, Richalet JP. Ventilatory and cardiac responses to hypoxia at submaximal exercise are independent of altitude and exercise intensity. J Appl Physiol 2012;112:566-70.

DOI: 10.1056/NEJMc1309747

TO THE EDITOR: Bärtsch and Swenson suggest that an age younger than 46 years is a risk factor for acute mountain sickness. This statement is not supported by data from controlled studies showing that the prevalence of acute mountain sickness after rapid ascent by train to $3500 \mathrm{~m}$ was roughly $50 \%$ lower in children than in adults. ${ }^{1,2}$ Moreover, and in contrast to a history of acute mountain sickness in adults, a history of this illness in children had no predictive value for recurrence of the condition during subsequent exposure; this suggests that a history of acute mountain sickness in children should not prompt practitioners to advise against reexposure or prescribe drugs for prophylaxis against this condition. ${ }^{2}$

The authors also recommend the use of tadalafil for prophylaxis against high-altitude pulmonary edema. This recommendation is basically sound, since defective nitric oxide synthesis underpins the susceptibility to this problem. ${ }^{3}$ However, in what is to our knowledge the only controlled study of tadalafil involving high-altitude climbers, this agent induced severe acute mountain sickness that necessitated descent in $20 \%$ of the participants. ${ }^{4}$ Tadalafil is not ideal for a prophylactic treatment, particularly since alternatives exist. ${ }^{3}$

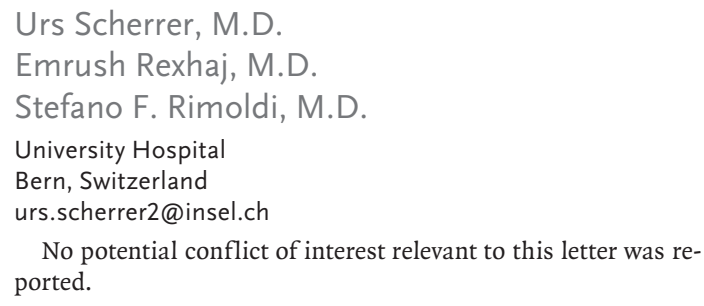

1. Bloch J, Duplain H, Rimoldi SF, et al. Prevalence and time course of acute mountain sickness in older children and adolescents after rapid ascent to 3450 meters. Pediatrics 2009;123:1-5. 2. Rexhaj E, Garcin S, Rimoldi SF, et al. Reproducibility of acute mountain sickness in children and adults: a prospective study. Pediatrics 2011;127(6):e1445-e1448.

3. Scherrer U, Allemann Y, Rexhaj E, Rimoldi SF, Sartori C. Mechanisms and drug therapy of pulmonary hypertension at high altitude. High Alt Med Biol 2013;14:126-33.

4. Maggiorini M, Brunner-La Rocca HP, Peth S, et al. Both tadalafil and dexamethasone may reduce the incidence of highaltitude pulmonary edema: a randomized trial. Ann Intern Med 2006;145:497-506.

DOI: 10.1056/NEJMc1309747

TO THE EDITOR: High-altitude retinal hemorrhage occurs frequently during mountain climbing because of hypoxic dilation of retinal veins and increased retinal venous pressure secondary to physical exertion and coughing. ${ }^{1}$ The prospective climber of Mount Kilimanjaro (5895 m) described in the article by Bärtsch and Swenson faces a considerable risk of high-altitude retinal hemorrhage, in addition to acute mountain sickness, high-altitude pulmonary edema, and highaltitude cerebral edema. ${ }^{2}$ In prospective studies involving high-altitude climbers, high-altitude retinal hemorrhage was observed in 60 to $74 \%$ of persons. ${ }^{2,3}$ This condition may occur either during the climb or after the descent. Although highaltitude retinal hemorrhage is frequently asymptomatic and resolves spontaneously, any decrease in visual acuity may signal extensive bleeding or macular involvement and should prompt immediate accompanied descent. ${ }^{4}$ Occasionally, permanent scotomata result. Unfortunately, neither slow acclimatization to high altitudes nor drug pro- 
phylaxis with the use of acetazolamide, glucocorticoids, or calcium-channel blockers prevents high-altitude retinal hemorrhage. This patient should be advised of the ocular hazards of his proposed ascent to high altitudes and should have an ophthalmologic examination after descent to identify resolving high-altitude retinal hemorrhage and any residual visual defect.

E. Sage Colombo, M.D., Ph.D.
University of New Mexico Hospital
Albuquerque, NM
ecolombo@salud.unm.edu
Irwin Hoffman, M.D.
Christus St. Vincent Hospital
Santa Fe, NM
$\quad$ No potential conflict of interest relevant to this letter was re-
ported.

1. Frayser R, Houston CS, Bryan AC, Rennie ID, Gray G. Retinal hemorrhage at high altitude. N Engl J Med 1970;282:1183-4.

2. Wiedman M, Tabin GC. High-altitude retinopathy and altitude illness. Ophthalmology 1999;106:1924-7.

3. Botella de Maglia J, Martínez-Costa R. High altitude retinal hemorrhages in the expeditions to 8,000 meter peaks: a study of 10 cases. Med Clin (Barc) 1998;110:457-61. (In Spanish.)

4. Bosch MM, Barthelmes D, Landau K. High altitude retinal hemorrhages — an update. High Alt Med Biol 2012;13:240-4.

DOI: 10.1056/NEJMc1309747

TO THE EDITOR: Bärtsch and Swenson conclude that the efficacy of acetazolamide in the prevention of acute mountain sickness during fast climbs is unknown. At least one excellent study ${ }^{1}$ on Mount Rainier clearly showed that $750 \mathrm{mg}$ of acetazolamide prevented acute mountain sickness during a rapid ascent of this mountain. Bärtsch and Swenson cite another study ${ }^{2}$ that showed that even a dose of $125 \mathrm{mg}$ twice daily prevented acute mountain sickness during a rapid ascent from 1600 to $4300 \mathrm{~m}$. They mention another study $^{3}$ of a Mount Kilimanjaro climb that did not show any efficacy of $500 \mathrm{mg}$ of acetazolamide in the prevention of acute mountain sickness, but it was not a randomized, controlled trial.

Finally, a marathon runner is featured in the case vignette. Anecdotally, in the Himalayas, trekkers who exercise strenuously at sea level often appear to be more prone to acute mountain sickness because they may also exert themselves at high altitudes, and such exertion at high altitudes may be counterproductive. ${ }^{4}$

Buddha Basnyat, M.D.

International Society for Mountain Medicine

Kathmandu, Nepal

rishibas@wlink.com.np
No potential conflict of interest relevant to this letter was reported.

1. Larson EB, Roach RC, Schoene RB, Hornbein TF. Acute mountain sickness and acetazolamide: clinical efficacy and effect on ventilation. JAMA 1982;248:328-32.

2. van Patot MC, Leadbetter G III, Keyes LE, Maakestad KM, Olson S, Hackett PH. Prophylactic low-dose acetazolamide reduces the incidence and severity of acute mountain sickness. High Alt Med Biol 2008;9:289-93.

3. Kayser B, Hulsebosch R, Bosch F. Low-dose acetylsalicylic acid analog and acetazolamide for prevention of acute mountain sickness. High Alt Med Biol 2008;9:15-23.

4. Richalet JP, Larmignat P, Poitrine E, Letournel M, CanouïPoitrine F. Physiological risk factors for severe high-altitude illness: a prospective cohort study. Am J Respir Crit Care Med 2012;185:192-8.

DOI: 10.1056/NEJMc1309747

THE AUTHORS REPLY: We are aware of the unique prospective study by Richalet and colleagues. However, their recommendations are based on the response of only one third of the tested persons, who returned self-reported information about a subsequent exposure to high altitudes. Selection bias due to a low response rate, lack of medical documentation of illness, and possible recall bias regarding medication and ascent rates limit the generalizability of the study by Richalet and colleagues. Furthermore, we consider a 7.3 percentage-point increase in prediction accuracy due to the addition of physiological measurements as being small. Moreover, this epidemiologic study does not address how to translate saturation, ventilation, and heart rate measured during hypoxic exercise into specific recommendations. Before proposing physiological assessments in advance of climbing, additional prospective data are needed to show that adding them to the approach summarized in our review improves the clinical outcome significantly.

The conclusion of Scherrer et al. that children are less susceptible to acute mountain sickness than adults is based on one study. In contrast, in two studies, reported prevalence rates of acute mountain sickness among children in various mountain areas were not appreciably different from those reported among adults in comparable settings, ${ }^{1,2}$ and in one direct comparison, the rates appeared higher among children than among adults. ${ }^{3}$ The poor reproducibility of acute mountain sickness in the study cited by Scherrer et al. could in part be explained by factors such as travel, anxiety, and disruption of daily routine to which particularly younger children are very susceptible (as shown by a report of a $21 \%$ fre- 
quency of "acute mountain sickness" at a sealevel summer camp). ${ }^{2}$ Finally, headache associated with tadalafil is one reason why we consider phosphodiesterase- 5 inhibitors as second-line drugs to the calcium-channel blocker nifedipine, which is cheaper and better evaluated for the prevention and treatment of high-altitude pulmonary edema.

We did not mention high-altitude retinal hemorrhages because, as Colombo and Hoffman state, they usually do not pose a health problem for climbers and their occurrence is not influenced by preventive measures for acute highaltitude illnesses. We agree with their recommendation for mountaineers, who have an acute loss of visual acuity at high altitudes.

The studies on acetazolamide cited by Basnyat do not address the situation of climbing rapidly to areas with very high altitudes such as Mount Kilimanjaro, since the final altitude in these studies was at most $4392 \mathrm{~m}$. Two studies cited in our review showed no correlation between maximal oxygen uptake and the development of acute mountain sickness; this suggests that other factors account for the anecdotal observations in climbers who are physically fit at sea level.

Peter Bärtsch, M.D.

University Hospital

Heidelberg, Germany

peter.bartsch@med.uni-heidelberg.de

Erik R. Swenson, M.D.

University of Washington

Seattle, WA

Since publication of their article, the authors report no further potential conflict of interest.

1. Dallimore J, Rowbotham EC. Incidence of acute mountain sickness in adolescents. Wilderness Environ Med 2009;20:221-4. 2. Theis MK, Honigman B, Yip R, McBride D, Houston CS, Moore LG. Acute mountain sickness in children at 2835 meters. Am J Dis Child 1993;147:143-5.

3. Moraga FA, Pedreros CP, Rodríguez CE. Acute mountain sickness in children and their parents after rapid ascent to $3500 \mathrm{~m}$ (Putre, Chile). Wilderness Environ Med 2008;19:287-92.

DOI: $10.1056 /$ NEJMc1309747

\section{Deficiency in Complement Factor B}

TO THE EDITOR: The alternative complement pathway is essential for defense against infection by polysaccharide-encapsulated bacteria. Factor B, factor $\mathrm{D}$, and properdin are required to stably initiate the process. ${ }^{1}$ Deficiencies of factor D and properdin have been described in humans. ${ }^{2-4}$ Here, we describe a 32-year-old woman with recurrent pneumococcal and meningococcal infection in whom factor B deficiency was detected.

The patient had nonconsanguineous parents of English and Scottish heritage. Her medical history revealed four clinically significant infections dating from childhood. At 2 years of age, she had primary pneumococcal peritonitis. Two years later, she was treated for communityacquired pneumonia. At 15 years of age, meningitis (caused by Neisseria meningitidis, serogroup Y) developed. At age 30 years, pneumococcal pneumonia complicated by a unilateral empyema developed. She required prolonged admission to the intensive care unit for type 1 respiratory failure that prompted suspicion of an immunodeficiency, and she underwent a thoracotomy to drain the empyema.

Screening tests revealed normal immunoglobulins and lymphocyte subsets. Classical comple- ment pathway activity was normal, but according to the results of a functional enzyme-linked immunosorbent assay (ELISA) (Wieslab, Euro Diagnostica), the alternative pathway was inactive.

Complement-mixing studies showed that the activity of the alternative pathway was restored to the patient's serum by properdin-deficient serum but not when the patient's serum was mixed with commercially sourced factor B-depleted serum. Factor B was undetectable by means of radial immunodiffusion (Binding Site) and ELISA ( $<36$ g per liter; reference range, 119 to 245).

Genome sequencing of all 18 coding exons of the CFB gene (transcript NM_001710.5) revealed compound heterozygous mutations that resulted in premature stop codons (protein truncation): a nonsense mutation (p.Q256X, c.766C $\rightarrow \mathrm{T}$ ) in exon 6 and a frameshift mutation (p.F632CfsX8, c.1894_1897delTTTG) in exon 15 (Fig. 1).

The patient's father was found to carry the c.1894_1897delTTTG mutation in exon 15. Her mother and sister carried the c.766C $\rightarrow \mathrm{T}$ mutation in exon 6. Her two children were both in excellent health; testing to ascertain which of the patient's mutations had been inherited by the children was deferred until they are older. Results of 